\title{
THE ORIGIN OF THE TERM "PNEUMONOKONIOSIS"
}

\section{INTRODUCED BY}

\section{A. MEIKLEJOHN}

\author{
From the Department of Industrial Health, University of Glasgow
}

(RECEIVED FOR PUBLICATION MARCH 10, 1958)

Throughout the literature on the dust diseases of the lungs, reference is frequently made to the fact that Professor F. A. Zenker of Erlangen introduced the term "pneumonokoniosis". As few authors have been able to study the original German article, this opportunity has been taken to present a translation in English prefaced by a short biographical note.

\begin{abstract}
Friedrich Albert von Zenker was born at Dresden on March 13, 1825. From 1843 to 1849 he studied medicine at Leipzig and Heidelberg and after graduation he went to Vienna to study pathology under Rokitansky and Hefohl. On his return to Germany he became professor of pathology and morbid anatomy at the University of Dresden. He undertook research into the pathology of the lungs, and in 1862 published his results entitled Beiträge zur normalen und pathologischen Anatomie der Lunge [Con-
\end{abstract}

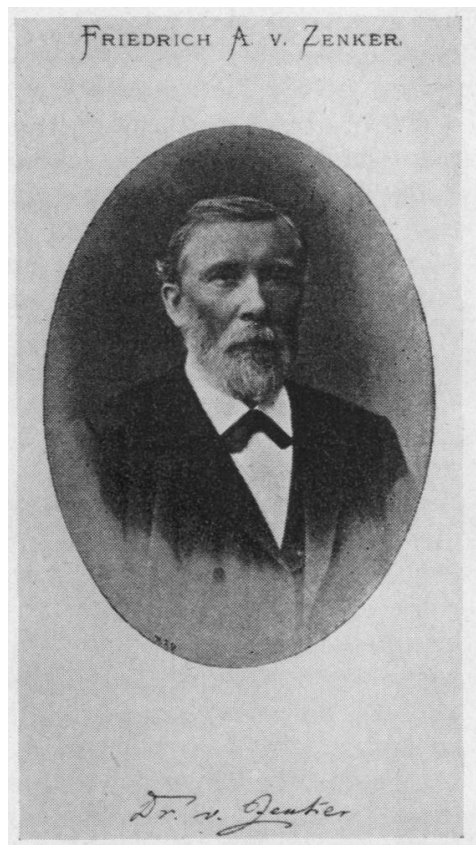

Friedrich Albert von Zenker (1825-1898). (Reproduced by courtesy of the Wellcome Historical Medical Museum.) tributions on the

Normal and Pathological Anatomy of the Lung]. In the same year he became professor of pathology at the University of Erlangen, where he remained until his death on June 13, 1898. At Erlangen he continued his studies of lung pathology, concentrating on the morbid changes associated with the inhalation of dust. It is in relation to the dust diseases of the lungs that his name endures, particularly because he invented the term "pneumonokoniosis".

Although references to this neologism occur frequently in the literature of the dust diseases of the lungs, few British authors have had the opportunity of studying the original article. My purpose is to make available, in translation, the relevant passages of the original article so that those interested in industrial medicine and others may learn the circumstances which led Zenker to coin the term. The article, entitled Ueber Staubinhalationskrankheiten der Lungen [Diseases of the Lungs due to the Inhalation of Dust] is a general review of current knowledge of the subject. It incorporates original observations on Die Eisenlunge [iron lung] or Siderosis pulmonum [pulmonary siderosis] (pp. 130-171). It is at the conclusion of this section that Zenker discusses nomenclature and suggests the generic term pneumonokoniosis. The manuscript was passed for publication in July, 1866, but the work was not published until 1867. These facts are mentioned because throughout the literature the reference is usually dated 1866 , when, in fact, it should be 1867 .

I am indebted to my colleague $H$. T. Betteridge, M.A., Ph.D., lecturer in German in the University of Glasgow, who edited my crude translation and assisted me cordially. The translation may appear rather literal but this is intentional, as I wished to reproduce the script as closely as possible.

\section{IRON LUNG-SIDEROSIS PULMONUM}

BY

\section{F. A. ZENKER}

I received the first specimen of this remarkable disease of the lungs on January 31, 1864, through the kindness of Dr. Geist, director of the medical department of the Nürnberg City Hospital, and his then assistant, Dr. Degen, to whom I am deeply indebted for sending numerous interesting specimens. It consisted of both lungs of a 31-year-old female factory worker, who was admitted to Nürnberg Hospital on January 19, 1864, on account 
of an illness alleged to be of six weeks' duration, and who died there on January 30 . In her work she had had much to do with red pigment. I can affirm that scarcely ever has the appearance of a pathological specimen so surprised me as that of these lungs. They showed throughout, on the surface as well as on section, a brick-red pigmentation so intense that all other (blood and tissue) colouring was almost entirely obscured by it, just as if the surfaces were smeared with red paint. Several large cavities and numerous firm fibrous nodules, the structure of which also was sprinkled with red spots, were scattered throughout both lungs. The peculiar nature of the pigmentation at a mere glance left no doubt that here we were not dealing with a morbid formation of pigmentation, but that the pigment must derive from a foreign colouring matter introduced from outside. At first I thought particularly of cinnabar or red lead. The chemical investigation, which Professor v. Gorup very kindly undertook at my request, and whose detailed finding I shall later mention, demonstrated that the colour was derived from iron oxide, which had accumulated in enormous quantity. Thus there no longer seemed to be any doubt about the connexion between the lung discoloration and her work with pigment. Histological investigation, however, showed that the ferrous material was deposited in the form of very fine particles, partly in the air passages, partly and very notably in the lung tissue itself, and even in the pulmonary pleura. The bronchial glands also displayed large red stains of iron. The iron particles had consequently penetrated into the interstices of the lung and had thence been distributed farther through the lymph channels. The whole state of affairs, especially in view of the large quantities of iron particles which were, as described, to be found in the air passages (bronchi and alveoli), seemed to permit no other explanation than that the iron material had reached the air passages through inhalation and been distributed from the alveoli into the lung parenchyma; that therefore the patient had worked with a dry pigment powder and that she must have been exposed for a very long period to the inhalation of a red colouring matter. To be certain of this I therefore asked Dr. Degen to make careful enquiries about the nature of the employment of the patient, about her whole mode of life and about the nature of the pigment with which she had worked; and at the same time to make enquiries whether any frequent attacks of illness, which could be attributed to a similar affection of the lungs, occurred among the workers in the same factory. Dr. Degen informed me of the result of his careful enquiries and also gave me a precise account of the illness and the results of the post- mortem examination and a description of the organs which had not been sent to me.

I will give this here in his own words and append the anatomico-histological description of the lungs which $\mathrm{I}$ received in toto.

Mary Frank, aged 31 years, factory worker from Steinbühl near Nürnberg, was admitted to Nürnberg Hospital on January 19, 1864.

\section{History}

As a child the patient was always healthy, although not particularly robust. About the age of 20 , before she started work in the factories described below, she worked for some years in a match factory at Nürnberg. There also she is said to have been, on the whole, well; at least she never complained of any chest trouble. She had several children, the last about two years ago; this child is still alive and perfectly healthy. Seven years ago she started work in one of the factories, where she remained until shortly before her death. Now as to these factories they were engaged in the manufacture of the little books of blotting paper in which fine gold-leaf is laid. The workplace consists usually of only two rooms; in the one the blotting paper was coloured and in the other the books were stapled together. About four girls were employed there. The place, where the colouring was done, is very small, about $8 \mathrm{ft}$. long, $5 \mathrm{ft}$. broad and $7 \mathrm{ft}$. high. There is no special system of ventilation; if the dust becomes too bad the windows are opened. If these were shut (and in two factories visited by Dr. Degen they were) the dust is fairly thick. Not only are the walls, benches, etc. red, but the atmosphere also seems coloured so that anyone who is not used to it feels his mouth dry within a few minutes. In the rooms where the binding is done, there is also dust. The air here, however, can be breathed without inconvenience. In these rooms work is carried on throughout the whole day and meals also are taken there, without the workers taking any special measures against the inhalation of dust. The operation of staining consists simply in applying the finely powdered pigment dry on to a sheet of blotting paper and rubbing it in with a piece of felt until the paper is thoroughly impregnated. The pigment is obtained from a Nürnberg mill (in Wöhrd-place-name).

None of the other female workers complained of any chest trouble (and Dr. Degen questioned not only those who were employed at the time but also others who had worked there for longer or shorter periods). Certainly their mouths are always full of dust and their sputum is usually stained red. However, in the evening there are facilities whereby 
the girls can clean their skin and mouth before they leave the workshop (facilities of which, according to unanimous report, the patient, who is said to have been unclean in her habits, made very little use). There seemed to be some doubt about a female patient, who was in the medical wing of the Nürnberg Hospital from January 12 until February 15,1864 , and who was discharged at her own request. This patient had a bad cough, breathlessness, and a high temperature, without the physical investigation providing an adequate explanation of these symptoms. She too had been employed for several years in staining but she had sometime previously (about one year) given up that work. On the other hand, there was another woman, who had been under treatment for a feverish inflammation of the stomach. She had worked with the pigment for 10 years and her respiratory organs were perfectly healthy. Also in the above-mentioned colour mill, where the dust is so thick that the workers appear as if they were painted red, no similar illness has so far been observed.

Frank [the deceased] worked in two of these factories and was employed mainly in the staining of the paper. Information about her state of health at this period is rather scant as she associated with few people. All agree in this point, that the patient, although she worked more than the others, lived extremely frugally and irregularly; for example, at lunch she ate apples and similar things.

The first signs of lung disease appeared about one-and-a-half years ago and are said to have been a persistent cough and shortness of breath. The patient treated herself a good deal with household remedies; from time to time she had medical advice but, although the doctors advised her to give up her work, she continued to work in the colouring shop until Christmas, 1863, when her respiratory disorder became so severe that she left the factory and stayed with friends until her admission to hospital (January 19, 1864).

\section{Present Condition}

The patient's state of nutrition is bad; complexion pale with a livid flush. Strength is satisfactory so that the patient was able to be up and about most of the time during the first half of her stay in hospital. The temperature is not raised; the pulse is rapid and weak. The brain and nervous system are normal. The patient has no headache nor any feeling of giddiness; she has full use of her limbs; sensation is also well maintained.

The patient's appetite is good; the bowels are always rather constipated. Occasionally she complains of slight pains in her body; menstruation is regular.
Breathing is rather difficult and short; on inspiration chest movements are equal on both sides of the chest, which is well developed. There is, however, some retraction of the left upper lobe and this persists even on deep inspiration. The cardiac impulse is weak but in normal position. Percussion of the chest reveals no difference between one area and others. On auscultation, in addition to vesicular breathing, from time to time sonorous rhonchi are heard. The air entry is rather diminished above and immediately below the clavicle. The area of heart and liver dulness is normal; the heart sounds are normal.

The sputum is fairly homogeneous, mucoid, and scanty. Throughout the mucus one observes here and there minute red streaks (these were regarded as specks of blood from the lung such as one is accustomed to find in the sputum). Cough is moderate and becomes more violent in the morning. Mist. sal. ammon.

During the succeeding days the patient's condition, both in her own opinion and in the judgment of those attending her, remained unchanged and gave no cause for apprehension; indeed the patient asserted that the medicine eased her, because the phlegm was looser. On January 24 she noticed that her feet suddenly began to swell, especially round the ankles. The swelling spread quickly to the legs and to some extent above the knees, but was quite painless. The skin was pale and on pressure deep pitting persisted. From this date the patient was confined to bed. Her complexion became more cyanosed and the dyspnoea also increased. She did not complain of pain anywhere. During the succeeding days she developed dulness on both sides of her chest; air entry was diminished and vocal fremitus increased. The dulness advanced only to the lower angle of the scapula. Ascites now developed but so far was not serious. The body temperature did not rise; the pulse became very weak and rapid. The digestion continued unaffected. Mentally she remained clear until her death. During her last days the sputum became thick and purulent. Death due to increasing dyspnoea occurred on January 30 , at 1.30 a.m.

\section{Necropsy}

Nine hours after death. Very emaciated cadaver with pale flabby musculature; advanced rigor mortis, slight hypostasis, dropsy of lower extremities; colour in general pale, lips bluish; abdomen distended.

Neck.-Frothy mucus present in the larynx and in the large bronchi. 
Cranium.-Meninges normal; brain pale, somewhat moister than usual; in the lateral ventricles a little clear serum. Arteries at the base delicate; cerebellum normal.

Thorax.-In both pleural sacs a moderate effusion of clear serum (for description of lungs see below). There was practically no serum in the pericardium. The heart was flabby: the right ventricle dilated; valves healthy. Immediately above the semilunar valves of the aorta there were several yellowish-white irregularities varying from the size of millet seed to that of peas. The lungs which were sent to me showed the following condition (see plate II). Both lungs are almost everywhere covered by false membrane and adhesions. The covering so formed was in places, particularly on the right anterior aspect, as much as $1 \frac{1}{2} \mathrm{~mm}$. thick. On removal of the covering the surface of the lung was quite uniformly of a very intense brick-red colour, save that here and there the boundaries of the lobules appeared as black lines on the red background. And even the fibrous covering on removal showed here and there extensive brick-red discoloration on the surface in contrast with the pleura.

In some areas, notably over the right middle lobe, the surface is irregular, coarsely nodular, partly due to projection of the individual lobules as a result of contracted lobular septa, partly from projection of some of the nodules in the lung tissue, which will be described below.

On section it is seen that the aerated lung tissue, which still existed, is everywhere uniformly stained a very intense brick-red colour. And indeed on close inspection the cut surface appears as a uniformly fine cellular red network, in which the lobular septa are in many places defined as fine linear ridges, similarly intense brick-red in colour, and enclosing large polygonal areas, so that one can recognize even with the naked eye that the red colour is mainly restricted to the lung tissue itself. Nevertheless the air passages also contain some of the colouring matter, which exudes as a dull brickred fluid on to the cut surface.

In the thus constituted tissue of all the lobes of both lungs there are scattered numerous roundish, in section, greyish-yellow fibrous nodules from about the size of a pinhead to that of a large round pea and even in some cases larger, more irregular and coarse. These nodules are mostly interspersed with smaller or larger brick-red patches, in part also, though less extensively, with black specks.

While many of these nodules on section everywhere appear solid, others show in the centre a very minute pinprick-like opening (some of the larger ones even show several), apparently the lumen of one of the finer bronchioles, though any connexion with the larger bronchi could not be demonstrated with certainty. The apex of the right lung is occupied by thickly packed uniform nodules; likewise the main part of the left apex, while another part of it is transformed into a black sclerotic mass about $4 \mathrm{~mm}$. broad, in which a few very fine brickred points are scattered. Nowhere in the lungs were there any recent tubercles.

Finally all lobes, with the exception of the right middle, contain both large and small irregular sinuous cavities. The largest of these are in the somewhat shrunken right lower lobe, the whole upper half of which is excavated. This cavity is traversed by several bridge-like strands and its wall is in part fairly smooth, being covered with a loose greyish-yellow membrane, in part covered with a necrotic and sometimes brick-red mass. In both apices below the fibrous areas already described and in the left lower lobe there are small cavities from the size of a hazelnut to the size of a large cherry, the ragged walls of which are covered by necrotic masses, some pale greyish-yellow, others brick-red in colour.

The walls of the larger arteries and veins within the lung parenchyma are unstained throughout. Also the larger bronchi, which mostly contained some brick-red mucus in their lumen, do not show the brick-red coloration either on the inside surface or in the thickness of the wall. On the other hand, confluent brick-red spots occur in the finest bronchioles, which can still be followed with the scissors (that is those which are not too small to be opened up with scissors). These spots, as the microscopical investigation of vertical sections shows, are formed by dense granular deposits in the deeper tissue layers of the bronchial wall, whereas the surface layer is quite unstained. The thickness and the other characteristics of the bronchial wall, in the case of all the branches which can be traced with scissors, are quite normal.

The bronchial glands at the root of both lungs are about normal size, in the central areas mainly black, while the superficial layer is everywhere stained an intense brick-red colour. To some extent brick-red spots are scattered through the black coloured areas.

[At this point Zenker records in detail the results of the chemical investigation of the lung tissues undertaken for him by Professor von GorupBesanez.]

Fifty-seven g. of lung tissue treated with hydrochloric acid yielded $0.828 \mathrm{~g}$. iron oxide, equivalent to $14.5 \mathrm{~g}$. iron oxide per $1,000 \mathrm{~g}$. lung tissue. The entire right lung weighed $780 \mathrm{~g}$. Only $400 \mathrm{~g}$. of left lung were available for weighing but whole 
weight was estimated as $720 \mathrm{~g}$., which makes the aggregate weight of the two lungs approximately $1,500 \mathrm{~g}$. This indicates a total iron oxide content of about 21-22 g. The specific gravity of the lung tissue (a) from which all air was thoroughly expelled compared with that of a healthy man, aged 40 years $(b)$, and with that of an old man $(c)$, each after immersion in spirit was (a) 1.065, (b) 1.015 , and (c) 1.0025 .

Professor von Gorup-Besanez also examined the powder which the deceased had handled in her occupation and found it to be identical with that recovered from the lung tissues.

Microscopical examination shows (Plate III, Fig. 2) that the pigmentation of the tissue is caused by more or less densely packed fine particles deposited in the parenchyma, which, as regards their size and other features, agree completely with the previously described iron particles constituting the granular matter in the air passages. These lie thickest, aggregated into compact opaque masses, and thus obliterating all tissue elements in the lobular septa and to a great extent also in the infundibular septa. The latter are in part distended into enormous thick bands and particularly the nodules frequently form irregular, thick, opaque masses, which in bulk exceed the area of one or even of several alveolar sections. Where the deposit is less dense the iron particles in many places lie embedded in the parenchyma aggregated into little roundish or linear concentrations, thus giving the appearance of being enclosed within cells; and this hypothesis gains plausibility (though in the case of most of the accumulations it cannot be proved directly) through the little spaces, corresponding to the site of the granule, which here and there appear in these accumulations.

In the pleura as well as in the tough nodules (to which I shall come back later) these granular accumulations are mostly spindle-shaped. The alveolar septa are also in part densely permeated by iron particles and in places they then form shapeless thick bands, completely obscuring and significantly narrowing the alveolar spaces. Here the iron infiltration extends right up to the margin of the alveolus. In other areas, however, only isolated iron particles are scattered quite sparsely in the otherwise normal tissue of the alveolar septa, while other areas are quite free altogether. The innermost layer of the alveolar wall particularly (except for the most intensely infiltrated areas) is in the main free from particles. Similarly this was so where they are visible in the case of the projecting capillaries. Epithelium was not present in the alveoli. Here and there smaller or larger irregular areas of black lung appear through the brown masses. However, in view of the patient's age there is too little rather than too much evidence of these [black areas].

The adventitia of the finer air passages mostly reveal a rather dense, opaque infiltration of iron, whereas the rest of the bronchial wall is free of it. Only in the finest branches which can be traced with the scissors, as already remarked, has the iron infiltration penetrated right into the deeper layers of the mucous coat. And here the adjacent mucous membrane in places shows coloured globules with radial markings (apparently phosphorus, iron oxide, and vivianite) about which, however, I cannot say whether they were already present in the fresh specimen. Sporadic instances of the same formation have been found in some other specimens. The sclerotic nodules in the lung tissue everywhere present the structure of a coarse fibrous connective tissue, which reveals itself (after treatment with acetic acid) as being permeated with very small predominantly spindle-shaped elements. These in some places contain distinct small nodules and in other areas where they are more widely distributed they are filled with unstained (apparently fatty) particles or finally in those parts (corresponding to the brick-red spots of the cut surface) with brown iron particles. Here and there right through such a nodule can be seen running for some distance the still intact wall of an apparently obliterated blood vessel. The structure of the connective tissue within the nodule reaches right up to the edge of the lacunae. These cannot therefore be identified with certainty, even microscopically, as cross sections of bronchi.

Accordingly we are certainly entitled to expect that further confirmation will not be lacking for the evidence here described, now that attention has been directed to it. For this new form of disease, I coin, by analogy with anthracosis (which designation will soon be adopted generally) the name siderosis of the lungs (from $\sigma \iota \delta \eta \rho o s$, iron) and one can then designate as phthisis siderotica the most serious ulcerative forms, as illustrated by our first case (red phthisis as opposed to black phthisis). After we have learned to recognize in anthracotic and siderotic lungs two varieties of the disease, which are identical in all essential points with respect to mode of origin and symptoms and differ only in the nature of the inhaled dust, we may subsequently expect that still other comparable diseases will be added (for example that due to the inhalation of tobacco dust as will be established in the next paper). It will then be necessary to embrace under a single title all these essentially identical forms of disease. Instead of the long-winded designation employed by me as the title of this article, which appears to me to express best the 
essence of the matter, the name Pneumonokoniosis (from rovis, dust) recommends itself. One would then require to distinguish the individual forms as pneumonokoniosis anthracotica, siderotica, etc. (or just briefly as anthracosis, siderosis).

\section{NOTE}

\section{Specimen of Original Lung at St. Thomas's Hospital, London}

Dr. Roland John, at present curator of the pathology museum at St. Thomas's Hospital, London, has provided the following interesting item of information.

"The specimen (3B.11.9-1840A) to which you refer in your letter of December 2 is still in the museum. It is, probably, a portion of the specimen referred to by Dr. Greenhow (Path. Soc. Trans., 20, p. 57). Dr. E. H. Greenhow, assistant physician to the Middlesex Hospital, read a paper before the
Pathological Society of London on April 20, 1869. In it he refers to two cases of iron oxide pigmentation of the lung published by Zenker and showed the Society a thin slice from the lung of a woman of 31 years, who had worked for seven years at the task of rubbing iron oxide dust into the paper of books made to contain gold-leaf. Dr. Greenhow mentioned that he was able to show this specimen to the Society by the courtesy of Dr. Wilson Fox, who was professor of clinical medicine at University College, London. From the foregoing it would appear that Professor Wilson Fox was in possession of a slice of this lung which he may or may not have received directly from Zenker, that he gave some or all of the specimen to Dr. Greenhow for the museum at the Middlesex Hospital, and that the latter institution gave the part we have to the museum at St. Thomas's."

\section{REFERENCE}

Zenker, F. A. (1867)). Dtsch. Arch. klin., 2, 116.

This volume is available in the Medical Library of the University of Birmingham and in the Library of the Royal Faculty of Physicians of Birmingham and in the Library of the Royal Faculty of Physicians
and Surgeons, Glasgow, and the Royal Society of Medicine, London. 